\section{SAFETY AND EFFICACY OF APROTININ UNDER CONDITIONS OF DEEP HYPOTHERMIA AND CIRCULATORY ARREST}

Aprotinin has been successfully used to reduce blood loss and blood product requirements in patients undergoing primary and reoperative cardiac operations. Its safety and efficacy during profound hypothermia and circulatory arrest have been questioned, however. A retrospective review compared 24 patients who received aprotinin during complex aortic procedures under profound hypothermia and circulatory arrest with 24 age-matched patients undergoing similar procedures without aprotinin. Activated clotting time was maintained at longer than $\mathbf{5 0 0}$ seconds (kaolin activating agent) or longer than 750 seconds (celite). We observed no statistically significant difference in the incidence of neurologic events $(p$ not significant) or myocardial infarctions ( $p$ not significant), and there was a trend toward reduced in-hospital mortality rate in aprotinin-treated patients. A higher incidence of postoperative renal dysfunction was encountered in aprotinin-treated patients. Aprotinin recipients had a significant reduction in requirements for postoperative homologous erythrocytes $(p=$ 0.01). We conclude that aprotinin may be safely and effectively used in patients undergoing deep hypothermia and circulatory arrest. (J THORAC Cardiovasc SURG 1995;110:1615-22)

Daniel J. Goldstein, MD, Carolyn M. DeRosa, BA, Linda B. Mongero, CCP, Alan D. Weinberg, MS, Robert E. Michler, MD, Eric A. Rose, MD, Mehmet C. Oz, MD, and Craig R. Smith, MD, New York, N.Y.
$A_{p}^{p}$ protinin (Trasylol), a bovine-derived nonspecific protease inhibitor, has been successfully used to decrease blood loss and blood product requirements in patients undergoing primary ${ }^{1,2}$ and reoperative $^{3,4}$ cardiac operations. Its mechanism of action has not yet been fully elucidated, but it is thought to reduce fibrinolysis, ${ }^{5,6}$ inhibit neutrophil activation, ${ }^{7}$ and preserve platelet function. 8,9

Patients undergoing complex aortic procedures performed with deep hypothermia and total circulatory arrest face a significant bleeding risk and would therefore be expected to benefit from the use of aprotinin. Two recent series reported an increased incidence of death, renal dysfunction, and multiorgan platelet thrombi in aprotinin recipients

From the Department of Surgery, Division of Cardiac Surgery, College of Physicians and Surgeons, Columbia University, New York.

Received for publication Jan. 6, 1995.

Accepted for publication May 9, 1995.

Address for reprints: Craig R. Smith, MD, Columbia Presbyterian Medical Center, 630 W. 168th St., Milstein Building, 7th floor, 7GN-435, New York, NY 10032.

Copyright (C) 1995 by Mosby-Year Book, Inc.

$0022-5223 / 95 \$ 5.00+0 \quad \mathbf{1 2} / \mathbf{1} / \mathbf{6 6 2 2 8}$ undergoing such procedures. ${ }^{10,11}$ An initial review of our experience did not support these findings. ${ }^{12}$ To address these issues, we completed a review of our institutional experience of complex aortic procedures performed with deep hypothermia $\left(18^{\circ} \mathrm{C}\right)$, circulatory arrest, and the use of aprotinin.

\section{Methods}

Between November 1, 1991, and June 31, 1994, 23 patients underwent complex aortic procedures and one patient underwent a renal tumorectomy under deep hypothermia and circulatory arrest with intraoperative use of aprotinin. Before this time, aprotinin was not used at our institution for this purpose. Twenty-four patients undergoing similar procedures without aprotinin during a more extended period (May 1, 1987, to December 31, 1993) were matched for age and type of operation to form a control group. The clinical, anesthesia, and perfusion records of all 48 patients were reviewed. Signed consent for aprotinin use was obtained for all treated patients under a protocol reviewed by the Institutional Review Board.

The treated group received the conventional "Hammersmith" (full dose) aprotinin regimen: (1) to assess the potential for an allergic reaction, a test dose of $1.4 \mathrm{mg}$ $(10,000 \mathrm{kIU})$ was given intravenously; (2) after induction of anesthesia but before sternotomy, a loading dose consisting of $280 \mathrm{mg}(2,000,000 \mathrm{kIU})$ was administered; (3) a pump prime dose of $280 \mathrm{mg}$ was added to the 
Table I. Clinical profile of aprotinin-treated and untreated (control) patients

\begin{tabular}{|c|c|c|}
\hline & Aprotinin & No aprotinin \\
\hline Number of cases & 24 & 24 \\
\hline \multicolumn{3}{|l|}{ Age $(y r)$} \\
\hline Mean $\pm \mathrm{SD}$ & $61.9 \pm 14$ & $62.8 \pm 12$ \\
\hline Range & $42-84$ & $31-80$ \\
\hline $\operatorname{Sex}(M / F)$ & $13 / 10^{*}$ & $16 / 8$ \\
\hline \multicolumn{3}{|l|}{ History } \\
\hline Previous sternotomy & $16.7 \%(4 / 24)$ & $4.2 \%(1 / 24)$ \\
\hline Diabetes mellitus & $0 \%(0 / 24)$ & $4.3 \%(1 / 23) \div$ \\
\hline Hypertension & $58.3 \%(14 / 24)$ & $59 \%(13 / 22) \S$ \\
\hline Preoperative renal insufficiencyt & $34.7 \%(8 / 24)$ & $45.8 \%(11 / 24)$ \\
\hline \multicolumn{3}{|l|}{ Principal diagnosis } \\
\hline Acute aortic dissection & 19 & 20 \\
\hline Thoracic aortic aneurysm & 3 & 3 \\
\hline Innominate stenosis & 1 & 0 \\
\hline $\begin{array}{l}\text { Renal tumor with inferior vena } \\
\text { cava invasion }\end{array}$ & 1 & 1 \\
\hline \multicolumn{3}{|l|}{ Secondary diagnosis } \\
\hline Aortic regurgitation & 6 & 7 \\
\hline Coronary artery disease & 4 & 1 \\
\hline Mitral regurgitation & 0 & 1 \\
\hline \multicolumn{3}{|l|}{ Primary surgical procedures } \\
\hline Repair of aortic dissection & 19 & 20 \\
\hline Replacement of aorta & 3 & 3 \\
\hline Aorta-innominate artery bypass & 1 & 0 \\
\hline Renal tumorectomy & 1 & 1 \\
\hline \multicolumn{3}{|l|}{ Secondary surgical procedures } \\
\hline Resuspension of aortic valve & 9 & 9 \\
\hline Aortic valve replacement & 2 & 3 \\
\hline Coronary artery bypass grafting & 4 & 1 \\
\hline Reimplantation of coronary artery & 3 & 1 \\
\hline Mitral valve replacement & 0 & 1 \\
\hline
\end{tabular}

"One patient underwent two operations.

$¥$ Serum creatinine level greater than $1.5 \mathrm{mg} / \mathrm{dl}$.

\$Data not available for one patient.

$\S$ Data not available for two patients.

cardiopulmonary bypass (CPB) circuit before institution of CPB; and (4) the loading dose was followed by a constant infusion of $70 \mathrm{mg} / \mathrm{hr}(250,000 \mathrm{kIU} / \mathrm{hr})$ until conclusion of the operation. Aprotinin and all other intravenous infusions were stopped during circulatory arrest and restarted after resumption of CPB. One patient in the treated group exposed to aprotinin during a previous operation was skin tested for aprotinin hypersensitivity, with negative results.

Initial anticoagulation consisted of $300 \mathrm{units} / \mathrm{kg}$ of intravenous heparin, with 4000 units added to the pump prime. Activated clotting time (ACT) was measured at the following points: (1) before CPB, (2) every 20 minutes during CPB, (3) immediately before circulatory arrest, (4) on restoration of $\mathrm{CPB}$, and (5) 10 minutes after protamine reversal. ACT was maintained at longer than 750 seconds (with celite activating agent) or longer than 500 seconds (with kaolin). Protamine dosage was determined by heparin-protamine titration assay and by measurement of heparin levels (Hepcon HMS; Medtronic Hemotech, Englewood, Colo.).

\section{Results}

Patient characteristics. Table I summarizes important demographic and clinical characteristics of both groups. The control group consisted of 24 patients with a mean ( \pm standard deviation [SD]) age of $62.8 \pm 12$ years. The aprotinin group comprised 23 patients, with one patient undergoing two aortic dissection repair procedures 105 days apart, for a total of 24 procedures. Mean age in the aprotinin group was $61.9 \pm 14$ years. The incidences of diabetes and hypertension were comparable in both groups, whereas the control group had a higher incidence of preoperative renal dysfunction (serum creatinine level $>1.5$ $\mathrm{mg} / \mathrm{dl}$ ). Four patients in the aprotinin group and one patient in the control group had undergone previous sternotomy. One patient in each group 
Table II. Intraoperative time variables for aprotinin-treated and untreated (control) patients

\begin{tabular}{lccc}
\hline & Aprotinin & No aprotinin & $\begin{array}{c}p \\
\text { Value }\end{array}$ \\
\hline CPB time (min) & & & 0.01 \\
Mean \pm SD & $183.1 \pm 65$ & $154.7 \pm 49$ & $100-274$ \\
Range & $106-359$ & & \\
Total circulatory arrest time (min) & $27.4 \pm 14$ & $30.1 \pm 13.7$ & $11-60$ \\
Mean \pm SD & $11-68$ & $74 \pm 43$ & 0.34 \\
Range & $100 \pm 66$ & $0-147$ & 0.07 \\
Aortic crossclamp time (min) & $0-231$ & & \\
Mean \pm SD & & \\
Range & &
\end{tabular}

Table III. Intraoperative heparin, protamine, and activated clotting time for aprotinin-treated and untreated (control) patients

\begin{tabular}{lccc} 
Parameter & Aprotinin & No aprotinin & $\begin{array}{c}p \\
\text { Value }\end{array}$ \\
\hline Heparin dose (units) & & $31771 \pm 8228$ & 0.01 \\
$\quad$ Mean \pm SD & $40187 \pm 12653$ & $21,000-64,500$ & \\
$\quad$ Range & $24,000-79,000$ & $332 \pm 111$ & 0.08 \\
Protamine dose (mg) & $276.5 \pm 85$ & $150-540$ & \\
$\quad$ Mean \pm SD & $130-450$ & $681 \pm 231$ & 0.003 \\
$\quad$ Range & $1073 \pm 385$ & $770 \pm 173$ & 0.004 \\
ACT (sec) & $1101 \pm 267$ & & \\
$\quad$ During CPB (mean \pm SD) & & \\
Before total circulatory & & \\
$\quad$ arrest (mean \pm SD)
\end{tabular}

had undergone pacemaker insertion. One patient in each group underwent resection of a renal tumor involving the inferior vena cava under hypothermic arrest. One patient in each group had a diagnosis of Marfan syndrome.

Intraoperative parameters. As depicted in Table II, aprotinin-treated patients had a longer CPB time than untreated patients, perhaps related to the greater fraction of patients in this group who had undergone previous sternotomy. Aortic crossclamp and total circulatory arrest times were similar in both groups. The longest CPB time (359 minutes) and longest aortic crossclamp time (231 minutes) were seen in a 67-year-old man with marfanoid features, who had a type A (DeBakey type I) aortic dissection 5 years after aortic valve replacement and required aortic root replacement with reimplantation of the coronary arteries. The longest circulatory arrest time (68 minutes) was necessary for a 67 -yearold woman with an acute type I aortic dissection, who underwent repair of the dissection, aortic valve resuspension, and quadruple coronary bypass. Both of these patients received aprotinin during operation.
Anticoagulation. The aprotinin-treated cohort received significantly higher doses of heparin compared with the untreated group $(p=0.01)$, but no such difference was observed in required protamine dosages (Table III). A statistically significant prolongation in ACT during CPB $(p=0.003)$ and immediately before circulatory arrest $(p=0.004)$ was achieved in the aprotinin-treated patients compared with the control group.

Bleeding parameters. Aprotinin recipients required less postoperative transfusion of exogenous packed erythrocytes than did control patients $(p=$ 0.01 ). The intraoperative blood product requirement and postoperative chest tube output (first 12 hours) were not markedly different between groups (Table IV). Four of 24 aprotinin recipients (16.7\%) received no exogenous products whatsoever, versus one of 24 patients $(4.1 \%)$ in the control group. Eleven patients $(45.8 \%)$ in the aprotinin group received less that 10 units of total blood products, compared with only three $(12.5 \%)$ in the untreated cohort. Mean preoperative, postoperative, and discharge hemoglobin and platelet counts were similar among both groups (Table V). 
Table IV. Comparison of preoperative and postoperative bleeding parameters in aprotinin-treated and untreated control patients

\begin{tabular}{|c|c|c|c|}
\hline Variable & Aprotinin\# & No aprotinin & $\begin{array}{c}p \\
\text { Value }\end{array}$ \\
\hline \multicolumn{4}{|l|}{ Transfusion requirements } \\
\hline $\begin{array}{l}\text { Exogenous packed erythrocytes transfused during operation } \\
\text { (units) }\end{array}$ & $3.4 \pm 0.6$ & $3.5 \pm 0.6$ & NS \\
\hline Other products* transfused during operation (units) & $11.3 \pm 2.6$ & $12.1 \pm 2.1$ & NS \\
\hline $\begin{array}{l}\text { Exogenous packed erythrocytes transfused after operation } \\
\text { (units) }\end{array}$ & $1.3 \pm 0.5 \dagger$ & $3.2 \pm 0.8$ & 0.01 \\
\hline Other products* transfused after operation (units) & $2.2 \pm 1$ & $6.5 \pm 2.8$ & NS \\
\hline Patients not requiring transfusion & $4 / 24(16.7 \%)$ & $1 / 24(4.1 \%)$ & NS \\
\hline Patients requiring $\leq 10$ total units of exogenous blood products & $11 / 24(45.8 \%)$ & $3 / 24(12.5 \%)$ & NS \\
\hline \multicolumn{4}{|l|}{ Chest tube output (ml) } \\
\hline First 12 hours & $488 \pm 92$ & $495 \pm 90$ & NS \\
\hline
\end{tabular}

Results are expressed as mean \pm standard error. $N S$, Not significant.

*Includes platelets, fresh-frozen plasma, and cryoprecipitate.

Table V. Hematologic parameters among aprotinintreated and untreated (control) patients

\begin{tabular}{lrr} 
& Aprotinin & \multicolumn{1}{c}{$\begin{array}{c}\text { No } \\
\text { aprotinin }\end{array}$} \\
\hline Hemoglobin (mg/dl) & & \\
$\quad$ Before operation & $11.8 \pm 0.3$ & $11.9 \pm 0.5$ \\
$\quad$ POD 3 & $9.8 \pm 0.3$ & $9.0 \pm 0.2$ \\
$\quad$ Before discharge & $10.4 \pm 0.4$ & $9.8 \pm 0.3$ \\
Platelet count (1000 cells/ml) & & \\
$\quad$ Before operation & $224 \pm 15$ & $263 \pm 21$ \\
$\quad$ At arrival at intensive care unit & $134 \pm 14$ & $134 \pm 15$ \\
$\quad$ Before discharge & $380 \pm 29$ & $402 \pm 45$ \\
\hline
\end{tabular}

Data are mean \pm standard error.

Adverse events. Complications are summarized in Table VI. No anaphylactic reactions were seen among patients receiving aprotinin. With regard to neurologic complications, no statistically significant difference was noted in the incidence of seizures $(p$ not significant), reversible neurologic deficits ( $p$ not significant), or cerebrovascular accidents ( $p$ not significant) between groups. Similarly, no statistically significant difference in the percentage of postoperative myocardial infarctions (new $Q$ waves) was noted ( $p$ not significant). Equal fractions of patients in each group required temporary postoperative dialysis (hemodialysis, continuous arteriovenous hemofiltration-dialysis, or continuous venovenous hemofiltration-dialysis), and none of the surviving patients in either group required permanent hemodialysis. Renal dysfunction, defined by Sundt and colleagues ${ }^{10}$ as a $50 \%$ elevation in serum creatinine above baseline level, was seen 3.5 times more frequently in the aprotinin group, but the difference did not reach statistical significance. Patients in either group with preoperative renal insufficiency (serum creatinine level $>1.5 \mathrm{mg} / \mathrm{dl}$ ) were not more likely to have postoperative renal dysfunction. No early deaths (within 2 weeks) were seen in the aprotinin-treated group, whereas four early deaths were recorded in the control group. The causes of death included left ventricular failure and persistent hypotension caused by intraoperative myocardial infarction (one), multisystem organ failure (one), ischemic bowel on postoperative day (POD) 4 (one), and Pseudomonas septicemia (one). Two late deaths (later than 2 weeks but before discharge) were seen in the aprotinin group. One patient died after sudden severe hypoxia caused by intrapulmonary hemorrhage on POD 17, and the second patient died on POD 20 from fulminant pneumonia and septicemia. The latter patient underwent autopsy, which confirmed the presence of bronchopneumonia with abscess formation. Blood cultures grew multiple species. No evidence was found of platelet-fibrin thrombi in the heart, kidneys, or other major vascular organs.

Three late deaths in the control group consisted of a sudden arrhythmia in one patient (POD 24), and progressive irreversible hypoxia in two patients (PODs 16 and 41). None of the control patients who died underwent postmortem examination.

\section{Discussion}

Clinical use of aprotinin in patients undergoing CPB has been shown to reduce blood loss and blood product requirements. ${ }^{1-4}$ Its safety and efficacy during hypothermic circulatory arrest has been controversial, however. Aprotinin appears to exert both anticoagulant and procoagulant effects. The inhibition of protein $\mathrm{C},{ }^{13}$ the kallikrein-kinin system, ${ }^{5}$ and 
Table VI. Adverse events occurring in patients undergoing hypothermic circulatory arrest with and without use of aprotinin

\begin{tabular}{|c|c|c|c|c|c|}
\hline \multirow[b]{2}{*}{ Adverse event } & \multicolumn{2}{|c|}{ Aprotinin } & \multicolumn{2}{|c|}{ No aprotinin } & \multirow[b]{2}{*}{$p^{*}$} \\
\hline & $n$ & $\%$ & $n$ & $\%$ & \\
\hline Allergic reaction or anaphylaxis & $0 / 24$ & 0 & - & NA & $\mathrm{NA}$ \\
\hline \multicolumn{6}{|l|}{ Neurologic events } \\
\hline Seizures & $0 / 24$ & 0 & $1 / 24$ & 4.2 & NS \\
\hline Reversible neurologic deficit & $1 / 24$ & 4.2 & $1 / 24$ & 4.2 & NS \\
\hline Stroke & $3 / 24$ & 12.5 & $3 / 24$ & 12.5 & NS \\
\hline Myocardial infarction & $0 / 24$ & 0 & $2 / 24$ & 8.3 & NS \\
\hline \multicolumn{6}{|l|}{ Renal dysfunction } \\
\hline Required dialysis & $2 / 24$ & 8.3 & $2 / 24$ & 8.3 & NS \\
\hline Elevation in serum creatinine level $>50 \%$ & $7 / 24$ & 29.2 & $2 / 24$ & 8.3 & NS \\
\hline In-hospital deaths & $2 / 24$ & 8.3 & $7 / 24$ & 29.2 & NS \\
\hline
\end{tabular}

$N A$, Not applicable; $N S$, not significant.

"McNemar's Test $\left(\chi^{2}\right)$ for paired data, with continuity correction.

$\alpha_{2}$-antiplasmin consumption ${ }^{14}$ translate into a prothrombotic state, whereas prolongations of the prothrombin time $\mathrm{e}^{15}$ and the activated partial thromboplastin time ${ }^{15,16}$ render aprotinin an anticoagulant. To predict events under hypothermic conditions, these seemingly opposing effects must be combined with the effects of hypothermia on fibrinolysis, platelet count and function, ${ }^{17}$ and clotting factor levels and activity. ${ }^{18}$ Because the summation of these effects is not easily quantified, the evaluation of the safety of aprotinin must be based on the outcome of clinical studies.

Sundt and associates ${ }^{10}$ administered aprotinin to 20 patients undergoing thoracic aortic procedures employing circulatory arrest and deep hypothermia, and compared the findings with findings in a wellmatched retrospective control group. Renal dysfunction, renal failure necessitating dialysis, and death were more frequent in the aprotinin group, and autopsies of these patients showed evidence of widespread platelet-fibrin thrombi. The adequacy of anticoagulation in patients receiving aprotinin has been questioned, however. ${ }^{12}$ The series was carried out before the distorting effects of aprotinin on celite ACT were recognized, ${ }^{16,19}$ which may explain why the aprotinin group received an average of 27,850 units of heparin versus 40,250 units in control patients, despite identical mean CPB times.

Westaby and associates ${ }^{11}$ reported their experience with 80 patients undergoing complex aortic procedures with and without perioperative aprotinin. They described an increased incidence of "bleeding and thrombosis related deaths" in aprotinin recipients. They acknowledged changing their heparinization protocol after about 2 years to ac- count for aprotinin's effect on celite ACT, but they provided no data relating results to time period or heparinization protocol. None of the data presented were subjected to statistical analysis. Our experience provides little support for the findings of Westaby and associates. ${ }^{11}$ We observed no statistically significant difference in the incidence of adverse neurologic events or myocardial infarctions, and we saw a trend toward reduced incidence of in-hospital deaths among aprotinin-treated patients. Aprotinintreated patients were benefited by a reduction in the requirement for postoperative erythrocytes and other blood products and by avoidance of the potential complications of blood-replacement therapy. At our institution, ACT was predominantly measured with a kaolin assay, which is essentially unaffected by aprotinin. ${ }^{20,21}$ In those cases in which celite was used as the activating agent, the ACT was maintained at more than 750 seconds.

We did observe trends in postoperative renal dysfunction similar to those reported by Sundt and associates. ${ }^{10}$ By POD 3, a higher incidence of renal dysfunction was encountered in aprotinin recipients. This finding did not reach statistical significance. At discharge from the hospital, serum creatinine level had returned to baseline in five of seven patients (71.4\%). In both groups, two patients underwent temporary continuous arteriovenous or venovenous hemodialysis for management of volume overload. None of the surviving patients required permanent hemodialysis.

The retrospective design of this study lends itself to the introduction of potential bias. The aprotinin group and the control group were partially concur- 
rent. This would appear to introduce the possibility of selection bias during the period of overlap, when some procedures were being done with aprotinin and some without. Aprotinin was first used at this institution by one surgeon (C. R. S.), and use was restricted to heart-lung transplants, double-lung transplants, and procedures in patients who had undergone multiple previous heart operations. On the basis of results in such patients, the same surgeon, who performed 30 of the 48 cases in both groups $(62.5 \%)$, started using aprotinin in all aortic dissections. The other three surgeons involved adopted use of aprotinin sequentially during the overlap period and used the drug uniformly thereafter. Although the possibility of selection bias can never be completely eliminated from a retrospective study, in this series treatment assignment by each surgeon was not based on patient characteristics.

Our results suggest that aprotinin may be safely and effectively used in patients undergoing deep hypothermia and circulatory arrest. Findings of other authors regarding incidence of adverse events are not substantiated by this study. In view of the limited data and the controversial nature of the available information, a randomized trial with a multicenter design (to ensure adequate number of patients and generalizable conclusions) is warranted to investigate the potential advantages (or disadvantages) of aprotinin use under conditions of hypothermia and circulatory arrest.

\section{REFERENCES}

1. Bidstrup BP, Royston D, Sapsford RN, Taylor KM. Reduction in blood loss and blood use after cardiopulmonary bypass with high dose aprotinin (Trasylol). J Thorac Cardiovasc Surg 1989;97:364-72.

2. Harder MP, Eijsman L, Roozendaal KJ, van Overen W, Wildevuur CRH. Aprotinin reduces intraoperative and postoperative blood loss in membrane oxygenator cardiopulmonary bypass. Ann Thorac Surg 1991;51:936-41.

3. Cosgrove DM, Heric B, Lytle BW, et al. Aprotinin therapy for reoperative myocardial revascularization: a placebo-controlled study. Ann Thorac Surg 1992;54: 1031-8.

4. Dietrich W, Barankay A, Hahnel C, Richter JA. High-dose aprotinin in cardiac surgery: three years' experience in 1,784 patients. $\mathbf{J}$ Cardiothorac Vasc Anesth 1992;6:324-7.

5. Fuhrer G, Gallimore MJ, Heller W, Hoffmeister HE. Aprotinin in cardiopulmonary bypass: effects on the Hageman factor (FXII)-kallikrein system and blood loss. Blood Coagul Fibrinolysis 1992;3:99-104.

6. Orchard MA, Goodchild CS, Prentice CRM, et al.
Aprotinin reduces cardiopulmonary bypass-induced blood loss and inhibits fibrinolysis without influencing platelets. Br J Haematol 1993;85:533-41.

7. Lord RA, Roath OS, Thompson JF, Chant ADB, Francis JL. Effect of aprotinin on neutrophil function after major vascular surgery. Br J Surg 1992;79:517-21.

8. Wildevuur CRH, Eijsman L, Roozendaal KJ, Harder MP, Chang M, van Overen W. Platelet preservation during cardiopulmonary bypass with aprotinin. Eur $\mathbf{J}$ Cardiothorac Surg 1989;3:533-8.

9. Van Overen W, Harder MP, Roozendaal KJ, Eijsman L, Wildevuur CRH. Aprotinin protects platelets against the initial effect of cardiopulmonary bypass. $\mathbf{J}$ Thorac Cardovasc SuRg 1990;99:788-97.

10. Sundt TM, Kouchoukos NT, Saffitz JE, Murphy SF, Wareing TH, Stahl DJ. Renal dysfunction and intravascular coagulation with aprotinin and hypothermic circulatory arrest. Ann Thorac Surg 1993; 55:1418-24.

11. Westaby S, Forni A, Dunning J, et al. Aprotinin and bleeding in profoundly hypothermic perfusion. Eur $\mathbf{J}$ Cardiothorac Surg 1994;8:82-6.

12. Smith CR, Mongero L, DeRosa CM, Michler RE, Oz MC. Safety of aprotinin in profound hypothermia and circulatory arrest. Ann Thorac Surg 1994;58:606-9.

13. Espana F, Estelles A, Griffin JH, Aznar J, Gilabert J. Aprotinin is a competitive inhibitor of activated protein C. Thromb Res 1989;56:751-6.

14. Blauhut B, Gross C, Necek S, Doran JE, Spath P, Lundsgaard-Hansen P. Effects of high-dose aprotinin on blood loss, platelet function, fibrinolysis, complement and renal function after cardiopulmonary bypass. J Thorac Cardiovasc Surg 1991;101:958-67.

15. Quereshi A, Lamont J, Burke P, Grace P, BouchierHayes D. Aprotinin: the ideal anticoagulant? Eur J Vasc Surg 1992;6:317-20.

16. Hunt BJ, Segal H, Yacoub M. Aprotinin and heparin monitoring during cardiopulmonary bypass. Circulation 1992;86;410-2.

17. Yoshihara $H$, Yamamoto $T$, Mihara H. Changes in coagulation and fibrinolysis occuring in dogs during hypothermia. Thromb Res 1985;37:503-12.

18. Reed RL, Bracey AW, Hudson JD, Miller TA, Fischer RP. Hypothermia and blood coagulation: dissociation between enzyme activity and clotting factor levels. Circ Shock 1990;32:141-52.

19. Hunt BJ, Segal H, Yacoub M. Guidelines for monitoring heparin by the activated clotting time when aprotinin is used during cardiopulmonary bypass [Letter]. J Thorac Cardiovasc Surg 1992;104:211-2.

20. Wang JS, Lin CY, Hung WT, Karp RB. Monitoring of heparin-induced anticoagulation with kaolin activated clotting time in cardiac surgical patients treated with aprotinin. Anesthesiology 1992;77:1080-4.

21. Wendel HP, Heller W, Gallimore MJ, Bantel $H$, Müller-Beissenhirtz $\mathrm{H}$, Hoffmeister HE. The pro- 
longed activated clotting time (ACT) with aprotinin depends on the type of activator used for measurement. Blood Coagul Fibrinolysis 1993;4:41-5.

\section{Commentary}

Coagulation abnormalities and bleeding commonly occur after profoundly hypothermic perfusion and circulatory arrest. This was an obvious area to test the potential benefits of aprotinin, and adverse clinical experience was reported early, when there was little guidance on intraoperative anticoagulant policy. ${ }^{1,2}$ The article from Goldstein and colleagues presents a contrary view of the hazards of aprotinin in profound hypothermia and claims benefits. The authors confirm an increased incidence of renal failure in aprotinin-treated patients but do not report multisystem failure from widespread platelet fibrin thrombi in the microvasculature. With the exception of anaphylaxis, most adverse effects of aprotinin have been attributed to deficiencies in the anticoagulant regimen. In reality, improved guidelines emerged only after reports of catastrophic events when early protocols were followed. ${ }^{3}$ The article of Goldstein and colleagues suggests yet another modification in anticoagulant policy, with ACT maintained at longer than 1000 seconds for patients undergoing hypothermic circulatory arrest.

When aprotinin was found to artifactually prolong celite ACT, the setting for inadequate anticoagulation became apparent. This situation should have been clearly defined before release of the drug in Europe and clinical trials in the United States. ACT is also inversely related to temperature. Although this effect is expressed equally in aprotinin-treated and control patients, it reduces the likelihood of reaching an ACT low enough to prompt further heparin administration. In the hypothermic patients described independently by Westaby and coworkers $^{1}$ and Sundt and associates, ${ }^{2}$ artifactual prolongation of ACT caused aprotinin-treated patients to receive less heparin than did control patients. With the benefit of this information, Goldstein and colleagues maintained celite ACT at longer than 750 seconds, or longer than 500 seconds with kaolin provocation, which is unaffected by aprotinin. In fact, patients treated with aprotinin received considerably more heparin and had ACTs longer than 1000 seconds, versus around 700 seconds for control patients. These are substantial differences between treatment and historical control patients, and the avoidance of disseminated intravascular coagulopathy supports the wisdom of this approach. Another difference between the Columbia and Oxford patients was duration of CPB. Oxford patients were maintained with a celite ACT longer than 700 seconds but had much shorter duration of CPB ( $<100$ minutes versus $>200$ minutes). Differences in the ability of platelets to aggregate may have played a role in the Oxford patients with type I dissection who had disseminated intravascular coagulation during circulatory arrest. Inhibition by aprotinin of the protein $C$ and kallikrein-kinin systems and of $\alpha_{2}$ antiplasmin consumption also predispose the patient toward a prothrombotic state during circulatory arrest., ${ }^{1,3}$

Hypothermia alone causes altered platelet morphology, with an increase in mean platelet volume, decreased enzyme function, altered arachadonic acid metabolism, and platelet sequestration in the hepatic sinusoids. ${ }^{4}$ This alteration leads to thrombocytopenia. Decreased ability to aggregate arises through reduced thromboxane release and the influence of plasmin-related degradation of glycoprotein Ib receptors. In normal circumstances (without aprotinin), thorough rewarming reverses impaired aggregation and depressed enzyme kinetics and is probably the single most important factor in the prevention of postoperative bleeding. 5,6 The influence of hypothermia on the clotting cascades is also complex. Hibernating amphibians have lower levels of clotting factors in the cold, which represents an adaptive response to prevent thrombosis during sluggish flow. Primates have lost this facility, apart from the kinetic slowing of enzyme activity during cooling. ${ }^{7}$ Consequently, the balance between thrombosis and fibrinolysis may be easily disturbed during hypothermic low-flow states and circulatory arrest. Cold-induced hypercoagulability may have contributed to widespread microvascular coagulopathy in the patients with thoracoabdominal aneurysms in the series of Sundt and coworkers. ${ }^{2}$ Bacterial translocation through ischemic gut can result in endotoxin release. ${ }^{8}$ This in turn stimulates the monocytes to produce thromboplastin and activate the extrinsic clotting pathway. The combination of cold-induced hypercoagulability with endotoxemia results in disseminated intravascular coagulation.

Does the study of Goldstein and colleagues really demonstrate benefit from aprotinin treatment? As with previous studies, there are methodologic weaknesses, particularly lack of randomization and the potential for bias. The authors admit that a randomized study design was not acceptable to the surgeons. This could be interpreted as bias in favor of aprotinin. In addition, the control group was not concurrent with the study group but overlapped it in time. Because the safety of aprotinin had been questioned, was the drug withheld from individuals considered to be at high risk for thromboembolic or nephrotoxic complications? There was no difference in intraoperative transfusion requirements for erythrocytes, platelets, fresh-frozen plasma, or cryoprecipitate. The authors did not state their threshold for blood transfusion, but there was no difference in chest tube drainage between the groups. Why then did patients with aprotinin require fewer blood products in the postoperative period?

In summary, this article continues the debate around the safety of aprotinin and suggests that prolongation of ACT longer than 1000 seconds may prevent the adverse effects described by Westaby and coworkers ${ }^{1}$ and Sundt and associates. ${ }^{2}$ Benefits from aprotinin in profound hypothermia and circulatory arrest are yet to be proved, and I agree with the authors that a prospective randomized trial is necessary. Meanwhile, my group uses aprotinin during the rewarming phase after circulatory arrest or by topical application or intravenous infusion at the end of the procedure if bleeding is a problem.

Stephen Westaby, MD

Department of Cardiothoracic Surgery Oxford Heart Center

John Radcliffe Hospital

Headington, OX3 9DU United Kingdom 


\section{REFERENCES}

1. Westaby S, Forni A, Dunning J, et al. Aprotinin and bleeding in profoundly hypothermic perfusion. Eur J Cardiothorac Surg 1994;8:82-6.

2. Sundt TM, Saffitz JE, Stahl DJ, Wareing TH, Kouchoukos NT. Renal dysfunction and intravascular coagulation after use of aprotinin in thoracic aortic operations employing hypothermic circulatory arrest. Ann Thorac Surg 1993;55:1418-24.

3. Westaby S. Aprotinin in perspective. Ann Thorac Surg 1993;55:1033-41.

4. Reed RL 2nd, Bracey AW, Hudson JD, Miller TA, Fischer RP. Hypothermia and blood coagulation: dissociation between enzymatic activity and clotting factor levels. Circ Shock 1990;32:141-52.
5. Valeri CR, Feingold H, Cassidy G, et al. Hypothermia induced reversible platelet dysfunction. Ann Surg 1987; 205:175-81.

6. Rohrer MJ, Natale AM. Effect of hypothermia on the coagulation cascade. Crit Care Med 1992;20:1402-5.

7. Ahmad N, Bube B, Agarwal GP, et al. Comparative studies of blood coagulation in hibernating and nonhibernating frogs. Thormb Haemost 1979;42:959-64.

8. Ferraro FJ, Spillert CR, Swan KG, Lazaro EJ. Coldinduced hypercoagulability in vitro. Am Surgeon 1992;58:355-7.

$12 / 1 / 68026$ 